\title{
A PERTURBATION-BASED MODEL FOR RECTIFIER CIRCUITS
}

\author{
VIPIN B. VATS AND H. PARTHASARATHY
}

Received 29 December 2005; Accepted 2 June 2006

A perturbation-theoretic analysis of rectifier circuits is presented. The governing differential equation of the half-wave rectifier with capacitor filter is analyzed by expanding the output voltage as a Taylor series with respect to an artificially introduced parameter in the nonlinearity of the diode characteristic as is done in quantum theory. The perturbation parameter introduced in the analysis is independent of the circuit components as compared to the method presented by multiple scales. The various terms appearing in the perturbation series are then modeled in the form of an equivalent circuit. This model is subsequently used in the analysis of full-wave rectifier. Matlab simulation results are included which confirm the validity of the theoretical formulations. Perturbation analysis acts a helpful tool in analyzing time-varying systems and chaotic systems.

Copyright (c) 2006 V. B. Vats and H. Parthasarathy. This is an open access article distributed under the Creative Commons Attribution License, which permits unrestricted use, distribution, and reproduction in any medium, provided the original work is properly cited.

\section{Introduction}

The rectification process plays a very important role in modern electronics, and rectifiers are widely used in power supply circuits. A simple half-wave rectifier contains a diode, a resistor, and a capacitor as shown in Figure 1.1. The governing differential equation of the half-wave rectifier can be obtained by writing the KCL at the output node for the circuit as shown in Figure 1.1,

$$
C \frac{d V_{0}}{d t}+\frac{V_{0}}{R}=I_{D}
$$

and where $I_{D}=I_{s}\left(V_{D} / V_{T}-1\right), V_{D}(t)=V_{i}(t)-V_{0}(t)$. In the above-mentioned equations $V_{i}$ is an input voltage, $I_{D}$ is the current flowing through the diode, and $V_{0}$ is the output voltage. If the piecewise linear model of diode is chosen, then the solution to the above 


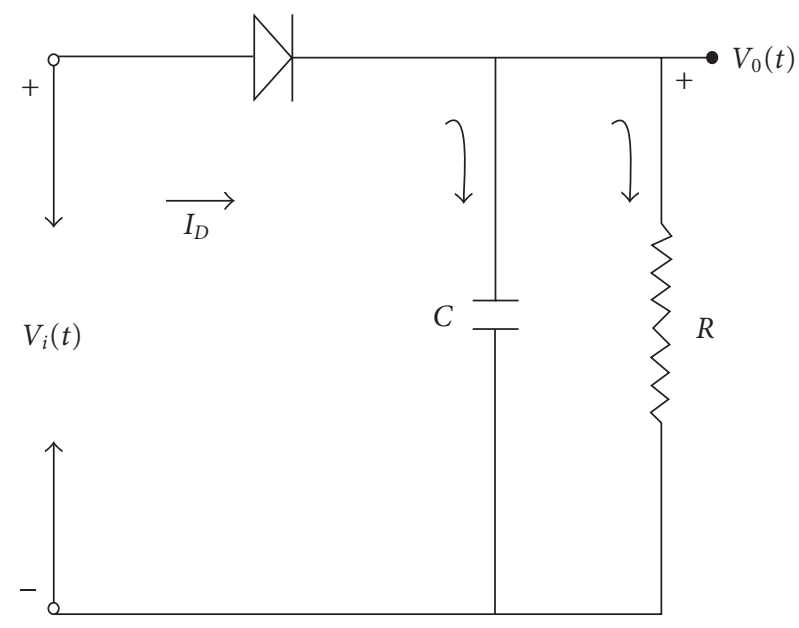

Figure 1.1. Half-wave rectifier circuit with capacitive load.

differential equation is possible in closed form, but for the nonlinear diode characteristics closed-form solution of the differential equation does not exist $[1,3,5]$. Such type of circuits have been studied in detail and many models have been proposed for the analysis of diode circuits.

In this paper, we had tried an alternative technique for analyzing the differential equation (1.1) through perturbation of theoretic methods. The perturbation technique used in this paper is a widely used method in quantum mechanics and celestial mechanics. In quantum mechanics, we seek a time-independent solution to the eigenvalue problem $\mathbf{H}\left|\psi_{\mathbf{n}}=E_{\mathbf{n}}\right| \psi_{\mathbf{n}}$. In such problems one expands the Hamiltonian $\mathbf{H}$ as $H=H_{0}+\epsilon H_{1}$, where $\epsilon H_{1}$ is considered as a small perturbation to $H_{0}[4]$ but $E_{n}$ and $\psi_{n}$ are also expanded as a power series of the same perturbation parameter in order to keep track of the variation. We use the perturbation technique for the rectifier circuit under the assumption that the diode has a weak nonlinearity. One of the techniques used in the analysis of such circuits is the method of multiple scales [2], in which the circuit parameters were used in deciding the smallness of the perturbation parameter $\epsilon$, but this may restrict the perturbation approach to certain circuits and one may not be able to generalize this method for all values of the parameter. However, in this paper, we overcome the limitation or dependence of the perturbation technique on the circuit parameters.

\section{Proposed nonlinear model of half-wave rectifier circuit using perturbation expansion}

In this section, we analyze the basic half-wave rectifier, as shown in Figure 1.1. Let the circuit be excited with a voltage $V_{i}$. We assume that the nonlinear diode characteristics are given by

$$
I_{D}=I_{s}\left(e^{V_{D} / V_{T}}-1\right)
$$


where $I_{s}$ is the reverse saturation current, $V_{D}$ is the terminal voltage across the diode, and $V_{T}$ is the temperature-dependent voltage. Plugging the above equation in (1.1) we get

$$
C \frac{d V_{0}}{d t}+\frac{V_{0}}{R}=I_{s}\left(e^{V_{D} / V_{T}}-1\right)
$$

where $V_{D}=V_{i}-V_{0}$, assuming that the capacitor connected in the half-wave rectifier is not initially charged. As per the standard asymptotic technique, the nonlinearity and the output voltage are expanded as a power series in the perturbation parameter $\epsilon$. The smallness of the perturbation parameter is independent of the circuit components like $R$ and $C$ and is assumed to be very small. This leads to

$$
V_{0}(t)=\sum_{n=0}^{\infty} \epsilon^{n} V_{0 n}(t)
$$

note that the diode characteristics can be expanded as

$$
f_{N L}\left(V_{D}\right)=I_{s}\left(e^{V_{D} / V_{T}}-1\right)=f_{L}\left(V_{D}\right)+\epsilon f_{N}\left(V_{D}\right),
$$

where $f_{L}\left(V_{D}\right)=I_{S} V_{D} / V_{T}$ and $f_{N}\left(V_{D}\right)=I_{S}\left(e^{V_{D} / V_{T}}-1-V_{D} / V_{T}\right)$. The term $f_{N}\left(V_{D}\right)$ is considered as a perturbation to the linear part of the characteristics $\left(f_{L}\left(V_{D}\right)\right)$ and the parameter $\epsilon$ is to be set equal to unity after completing all computations [4]. Plugging (2.3) and (2.4) in (2.2) we get

$$
\frac{d\left(\sum_{n} \epsilon^{n} V_{0 n}(t)\right)}{d t}+\frac{\sum_{n} \epsilon^{n} V_{0 n}(t)}{R C}=\frac{I_{s}}{C}\left(\frac{V_{D}}{V_{T}}+\epsilon\left(e^{V_{D} / V_{T}}-1-\frac{V_{D}}{V_{T}}\right)\right) .
$$

Now equating the coefficients of $\epsilon^{n}$ for $n=0,1,2$ results into three differential equations which are

$$
\begin{gathered}
\frac{d V_{o o}(t)}{d t}+\frac{V_{o o}(t)}{R^{\prime} C}=\frac{I_{S}}{C V_{T}} V_{i}(t), \\
\frac{d V_{o 1}(t)}{d t}+\frac{V_{o 1}(t)}{R^{\prime} C}=\frac{I_{S}}{C} g\left(V_{i}(t)-V_{o o}(t)\right), \\
\frac{d V_{o 2}(t)}{d t}+\frac{V_{o 2}(t)}{R^{\prime} C}=\frac{-I_{S}}{C} g^{\prime}\left(V_{i}(t)-V_{o o}(t)\right) V_{o 1}(t),
\end{gathered}
$$

where $g(x)=\exp \left(x / V_{T}\right)-1-x / V_{T}$ and $1 / R^{\prime}=1 / R+I_{S} / V_{T}$ (see [2]). The solutions to these differential equations are, respectively, given by

$$
\begin{gathered}
V_{00}=\frac{I_{s}}{C V_{T}} \int_{0}^{t} e^{-(t-\tau) / R^{\prime} C} V_{i}(\tau) d \tau, \\
V_{01}=\frac{I_{s}}{C} \int_{0}^{t} e^{-(t-\tau) / R^{\prime} C} g\left(V_{i}(\tau)-V_{00}(\tau)\right) d \tau, \\
V_{02}=\frac{-I_{s}}{C} \int_{0}^{t} e^{-(t-\tau) / R^{\prime} C} g^{\prime}\left(V_{i}(\tau)-V_{00}(\tau)\right) V_{01}(\tau) d \tau .
\end{gathered}
$$


4 A perturbation-based model for rectifier circuits

The calculations for the above equations were carried for sinusoidal input voltage $V_{i}=$ $V_{m} \sin (\omega t)$, whereas the method is applicable for any input voltage,

$$
V_{00}(t)=\frac{V_{m} I_{s}}{C V_{T} r}(\sin (\omega t-\phi))
$$

where $1 / R^{\prime} C=r \cos (\phi)$ and $\omega=r \sin (\phi)$. Plugging the above solution into the next integral, we can obtain the solution for the next perturbation:

$$
\begin{aligned}
V_{01}= & \frac{I_{s} \exp (-a t)}{C} \sum_{n=\text { odd }}^{\infty} \frac{R_{1}^{\prime n}}{n !(2)^{n}} \sum_{r=0}^{n}\left(\begin{array}{l}
n \\
r
\end{array}\right) \frac{(j)^{n-2 r+1}}{a^{2}+\omega^{2}(2 r-n)^{2}} A(t) \\
& +\frac{I_{s} \exp (-a t)}{C} \sum_{n=\text { even }}^{\infty} \frac{R_{1}^{\prime n}}{n !(2)^{n}} \sum_{r=0}^{n}\left(\begin{array}{l}
n \\
r
\end{array}\right) \frac{(j)^{n-2 r}}{a^{2}+\omega^{2}(2 r-n)^{2}} B(t),
\end{aligned}
$$

where $A(t)=e^{a t} f(\psi(t))-f(\xi), B(t)=e^{a t} g(\psi(t))-g(\xi), a=1 / R^{\prime} C, \psi(\tau)=(2 r-n)(\omega \tau+$ $\theta)$, and $\xi=(2 r-n) \theta$. For large " $t$," we neglect $e^{-t / R^{\prime} C}$ and thus obtain the steady-state solution in first-order perturbation terms. On the same lines, we can derive the secondorder perturbation term by evaluating the third integral given below:

$$
V_{02}=\frac{-I_{s}}{C} \int_{0}^{t} e^{-(t-\tau) / R^{\prime} C} g^{\prime}\left(V_{i}(\tau)-V_{00}(\tau)\right) V_{01}(\tau) d \tau .
$$

Rewriting the above integral in the following form we get

$$
V_{02}=\frac{-I_{s} \exp (-a t)}{C} \omega R_{1}^{\prime}\left[I_{1}+I_{2}-I_{3}\right]
$$

where

$$
\begin{gathered}
I_{1}=\int_{0}^{t} \frac{1}{2} \exp \left(a \tau+R_{1}^{\prime} \sin (\omega \tau+\theta)+j(\omega \tau+\theta)\right) V_{01}(\tau) d \tau \\
I_{2}=\int_{0}^{t} \frac{1}{2} \exp \left(a \tau+R_{1}^{\prime} \sin (\omega \tau+\theta)-j(\omega \tau+\theta)\right) V_{01}(\tau) d \tau \\
I_{3}=\int_{0}^{t} \exp (a \tau) \cos (\omega \tau+\theta) V_{01}(\tau),
\end{gathered}
$$

evaluating the integrals $I_{1}, I_{2}$, and $I_{3}$ gives the second-order perturbation term of voltage.

\section{Simulation results}

Simulation was performed using Matlab for the perturbation terms of a half-wave rectifier by discretizing the differential equation of each perturbation term. The result obtained, for the zeroth-order perturbation, was a sine wave with a very low magnitude. 


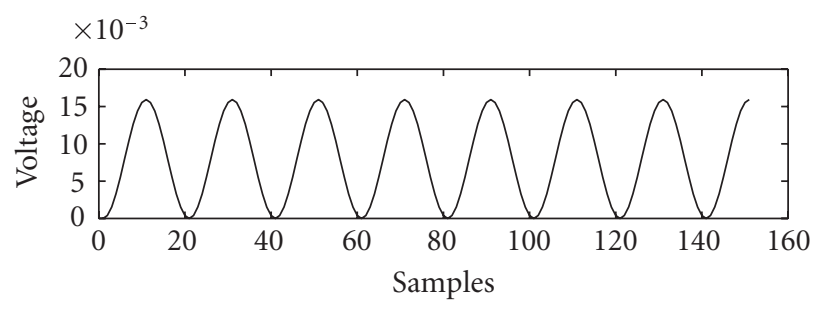

(a)

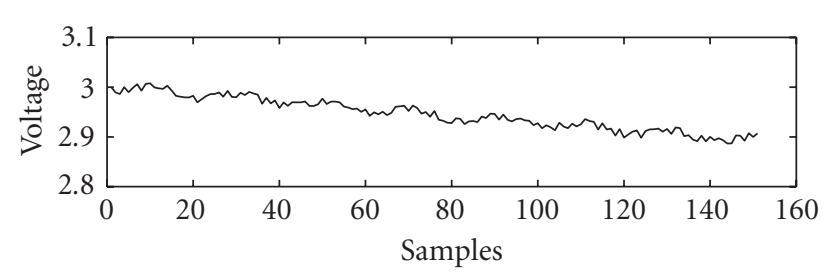

(b)

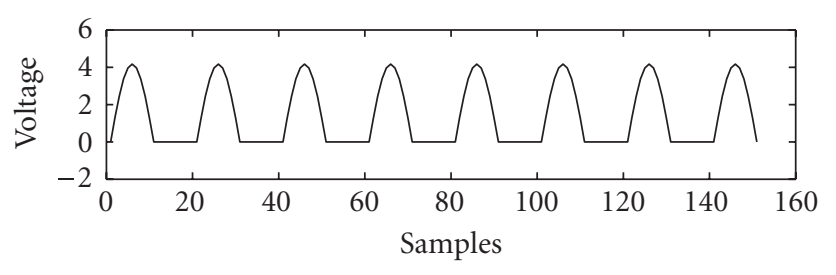

(c)

Figure 3.1. Perturbation result for half-wave rectifier: top most figure shows the zeroth-order perturbation followed by the first-order and the second-order, respectively.

This result can be thought as passing the sinusoidal input wave $V_{i}=V_{m} \sin (\omega t)$ through a series resistance $r_{n}=V_{T} / I_{s}$ in series with the load impedance ( $R$ and $C$ in parallel). Thus, diode in the rectifier acts as a linear resistance depending on the thermal voltage and the reverse saturation current. In the first-order perturbation the input to the circuit can be thought as $g(t)=e^{\left(V_{i}-V_{o o}\right) /\left(V_{T}\right)}-1-\left(V_{i}-V_{o o}\right) /\left(V_{T}\right)$, passing through the series combination of $r_{n}$ and the load impedance. Thus in this case the circuits remain the same except for the memoryless nonlinearity $g(t)$ which depends on the zeroth-order perturbation voltage. The wave form obtained in the output of the first-order perturbation term indicates that the voltage $g(t)=e^{\left(V_{i}-V_{o o}\right) /\left(V_{T}\right)}-1-\left(V_{i}-V_{o o}\right) /\left(V_{T}\right)$ is being traced at its envelope. Similar to the first-order perturbation we can formulate the model for the second-order perturbation by replacing $g(t)$ with $g^{\prime}(t)$. The output of the perturbation analysis of half-wave rectifier is shown in Figure 3.1. The model proposed using the half-wave rectifier circuits were used in the analysis of the full-wave rectifier and the results are shown in Figure 3.2. The proposed models of all the perturbation terms are shown in Figures 3.3, 3.4, and 3.5. 


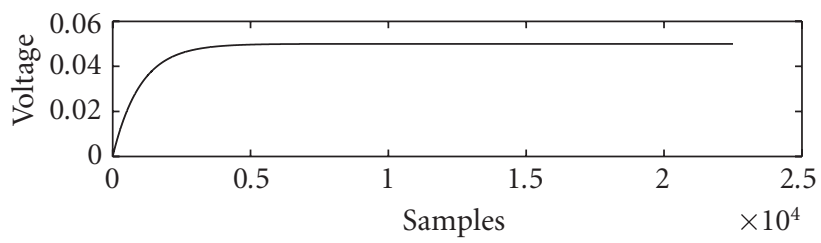

(a)

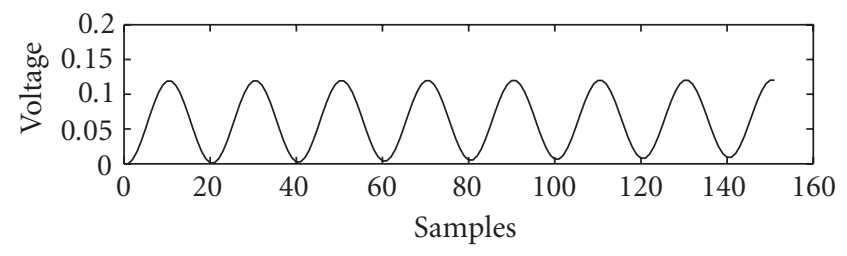

(b)

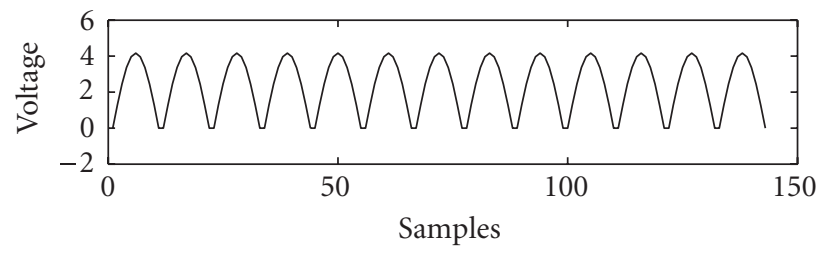

(c)

Figure 3.2. Results for the full-wave rectifier.

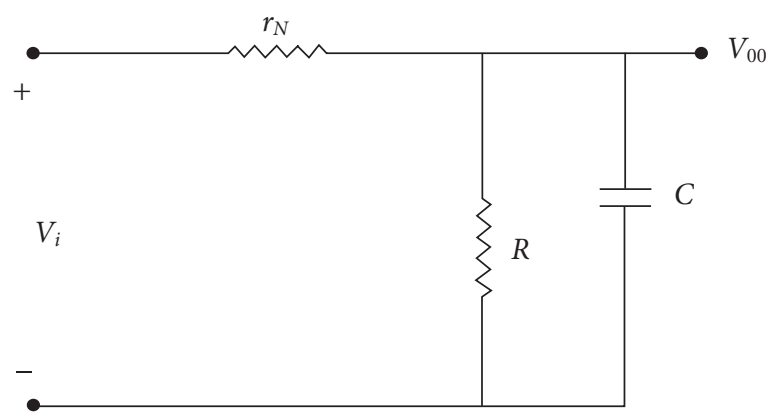

Figure 3.3. Zeroth-order perturbation model for diode in half-wave rectifier.

\section{Conclusion}

In this paper we have analyzed the half-wave rectifier circuit using the perturbation technique. The results obtained from each perturbation was finally transformed into an equivalent circuit model. With the help of these circuits, exact nonlinear analysis of rectifier 


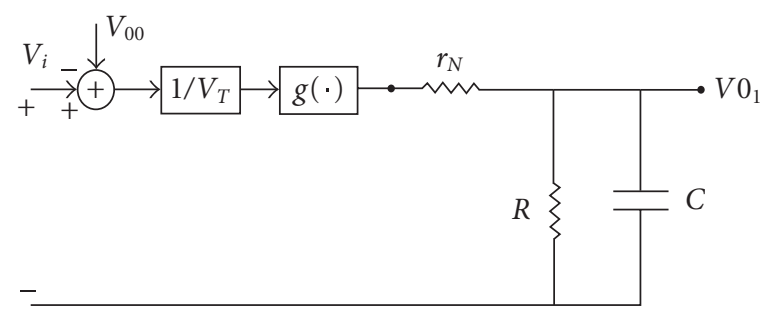

Figure 3.4. First-order perturbation model for diode in half-wave rectifier.

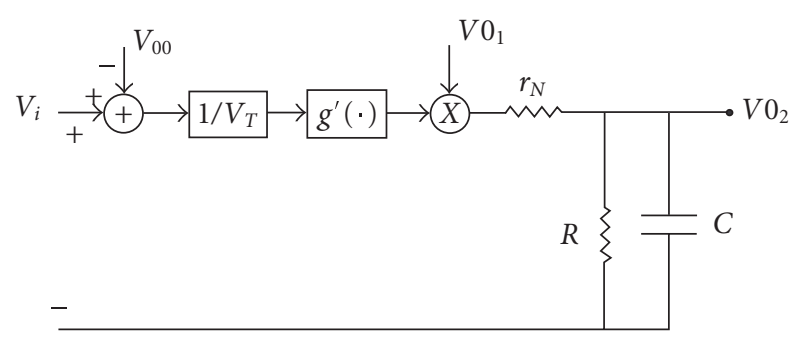

Figure 3.5. Second-order perturbation model for diode in half-wave rectifier.

circuits can be carried out. The model obtained for the zeroth-order perturbation stage resulted in a resistance defined as $r_{n}=V_{T} / I_{s}$. Using this structure for a diode, we have modeled the other two perturbations. To the author's best knowledge, such a model has never been proposed. The second-order perturbation model results, as shown in the above section, return nearly the exact rectified output. Further evaluation of the perturbation terms will lead to more detailed results. Equivalent model of the diode obtained in the zeroth-order perturbation is proposed to be as a resistance, $r_{n}=V_{T} / I_{s}$, which has never been proposed in the literature to the author's best knowledge thus revealing that the nonlinear behavior of diode will change with any change in temperature due to dependence on $V_{T}$.

\section{Appendices}

The following notation will be used in the derivations of the perturbation terms:

(A) $\exp (\tau)$ instead of $e^{\tau}$,

(B) Imag $(Z)$ represent the imaginary part of variable $Z$.

\section{A. Calculation of the zeroth-order perturbation term $V_{00}$}

$$
V_{00}=\frac{I_{s}}{C V_{T}} \int_{0}^{t} \exp \left(\frac{-(t-\tau)}{R^{\prime} C}\right) V_{i}(\tau) d \tau
$$


8 A perturbation-based model for rectifier circuits

Assuming $V_{i}=V_{m} \sin (\omega t)$,

$$
\begin{aligned}
V_{00}(t) & =\frac{I_{s} V_{m}}{C V_{T}} \int_{0}^{t} \exp \left(\frac{t-\tau}{R^{\prime} C}\right) \sin (\omega t) d \tau \\
& =\frac{I_{s} V_{m}}{C V_{T}} \exp \left(\frac{-t}{R^{\prime} C}\right) \operatorname{Imag}\left(\int_{0}^{t} \exp \left(\frac{\tau}{R^{\prime} C}\right) \exp (j \omega \tau) d \tau\right) \\
& =\frac{I_{s} V_{m}}{C V_{T}} \exp \left(\frac{-t}{R^{\prime} C}\right) \operatorname{Imag}\left(\frac{\exp \left(j \omega t+t / R^{\prime} C\right)-1}{1 / R^{\prime} C+j \omega}\right) \\
& =\frac{I_{s} V_{m}}{C V_{T}} \operatorname{Imag}\left(\frac{\exp (j \omega t)-\exp \left(-t / R^{\prime} C\right)}{1 / R^{\prime} C+j \omega}\right) .
\end{aligned}
$$

For large $t$, we neglect $\exp \left(-t / R^{\prime} C\right)$ and obtain the steady state solution as

$$
\begin{gathered}
V_{00}(t)=\frac{I_{s} V_{m}}{C V_{T}} \operatorname{Imag}\left(\frac{\exp (j \omega t)}{1 / R^{\prime} C+j \omega}\right), \\
V_{00}(t)=\frac{I_{s} V_{m}}{C V_{T} r} \sin (\omega t-\phi),
\end{gathered}
$$

where $r \exp (j \phi)=1 / R^{\prime} C+j \omega$.

\section{B. Calculation of the first-order perturbation term $V_{01}$}

$$
V_{01}=\frac{I_{s}}{C} \int_{0}^{t} \exp \left(\frac{-(t-\tau)}{R^{\prime} C}\right) g\left(V_{i}(\tau)-V_{00}(\tau)\right) d \tau
$$

Let

$$
V_{m} \sin (\omega t)-V_{\text {om }} \sin (w t-\phi)=V_{T} R_{1}^{\prime} \sin (\omega t+\theta),
$$

where

$$
\begin{gathered}
V_{\mathrm{om}}=\frac{V_{m} I_{s}}{C V_{T} r} \\
R_{1}^{\prime}=\sqrt{\left(\left(V_{\mathrm{om}} \sin (\phi)\right)^{2}+\left(V_{m}-V_{\mathrm{om}} \cos (\phi)\right)^{2}\right) / V_{T}} \\
\theta=\tan ^{-1}\left(\frac{V_{\mathrm{om}} \sin (\phi)}{V_{m}-V_{\mathrm{om}} \cos (\phi)}\right), \\
V_{01}(t)=\frac{I_{s}}{C} \int_{0}^{t} \exp \left(\frac{\tau-t}{R^{\prime} C}\right)\left(\exp \left(R_{1}^{\prime} \sin (\omega \tau+\theta)\right)-1-R_{1}^{\prime} \sin (\omega \tau+\theta)\right) d \tau .
\end{gathered}
$$

Simplifying the second term in the integral we get

$$
\exp \left(\frac{V_{i}-V_{o o}}{V_{T}}\right)-1-\frac{V_{i}-V_{o o}}{V_{T}}=\sum_{n=2}^{\infty} \frac{1}{n !} R_{1}^{n} \sin ^{n}(\omega \tau+\theta) .
$$


Since we have

$$
\sin ^{n}(x)=\left(\frac{\exp (j x)-\exp (-j x)}{2 j}\right)^{n}=\frac{1}{(2 j)^{n}} \sum_{r=0}^{n} C_{r}^{n} \exp (j(2 r-n) x)(-1)^{r-n}
$$

we get

$$
\begin{aligned}
\exp ( & \left.\frac{V_{i}-V_{o o}}{V_{T}}\right)-1-\frac{V_{i}-V_{o o}}{V_{T}} \\
& =\sum_{n=2}^{\infty} \frac{R_{1}^{\prime n}}{n !(2 j)^{n}} \sum_{r=0}^{n}\left(\begin{array}{l}
n \\
r
\end{array}\right)(-1)^{n-r} \exp (j(2 r-n)(\omega \tau+\theta)),
\end{aligned}
$$

therefore the net integral results into

$$
=\frac{I_{s} \exp \left(-t / R^{\prime} C\right)}{C} \sum_{n=2}^{\infty} \frac{R_{1}^{\prime n}}{n !(2 j)^{n}} \sum_{r=0}^{n}\left(\begin{array}{l}
n \\
r
\end{array}\right)(-1)^{n-r} \int_{0}^{t} \exp \left(j(2 r-n)(\omega \tau+\theta)+\frac{\tau}{R^{\prime} C}\right) d \tau,
$$

thus we get the final solution to the first-order perturbation terms as the real part of the following equation:

$$
\begin{aligned}
= & \frac{I_{s} \exp \left(-t / R^{\prime} C\right)}{C} \sum_{n=2}^{\infty} \frac{R_{1}^{\prime n}}{n !(2 j)^{n}} \sum_{r=0}^{n}\left(\begin{array}{l}
n \\
r
\end{array}\right)(-1)^{n-r}\left[\frac{\exp \left(j(2 r-n)(\omega \tau+\theta)+\tau / R^{\prime} C\right)}{1 / R^{\prime} C+j(2 r-n) \omega}\right]_{0}^{t} \\
= & \frac{I_{s} \exp \left(-t / R^{\prime} C\right)}{C} \sum_{n=2}^{\infty} \frac{R_{1}^{\prime n}}{n !(2 j)^{n}} \sum_{r=0}^{n}\left(\begin{array}{l}
n \\
r
\end{array}\right)(-1)^{n-r} \\
& \times\left[\frac{\exp \left(j(2 r-n)(\omega t+\theta)+t / R^{\prime} C\right)}{1 / R^{\prime} C+j(2 r-n) \omega}-\frac{\exp (j(2 r-n) \theta)}{1 / R^{\prime} C+j(2 r-n) \omega}\right] .
\end{aligned}
$$

Real part of the above equation can be expressed in the form

$$
\begin{gathered}
V_{01}=V_{01, n=\text { odd }}+V_{01, n=\mathrm{even}} \\
V_{01}=\frac{I_{s} \exp (-a t)}{C} \sum_{n=\text { odd }}^{\infty} \frac{R_{1}^{\prime n}}{n !(2)^{n}} \sum_{r=0}^{n}\left(\begin{array}{l}
n \\
r
\end{array}\right) \frac{(j)^{n-2 r+1}}{a^{2}+\omega^{2}(2 r-n)^{2}}(\exp (a t) f(\psi)-f(\xi)) \\
+\frac{I_{s} \exp (-a t)}{C} \sum_{n=\text { even }}^{\infty} \frac{R_{1}^{\prime n}}{n !(2)^{n}} \sum_{r=0}^{n}\left(\begin{array}{l}
n \\
r
\end{array}\right) \frac{(j)^{n-2 r}}{a^{2}+\omega^{2}(2 r-n)^{2}}(\exp (a t) g(\psi)-g(\xi)),
\end{gathered}
$$

where $a=1 / R^{\prime} C, \psi(\tau)=(2 r-n)(\omega \tau+\theta), \xi=(2 r-n) \theta, g(\psi)=a \cos (\psi)+\omega(2 r-n) \sin (\psi)$, $f(\psi)=a \sin (\psi)-\omega(2 r-n) \cos (\psi)$. 
For the steady state solution the terms containing $e^{-a t}$ are neglected. Thus the steady state solution of $V_{01}$ is given by

$$
\begin{aligned}
V_{01}= & \frac{I_{s}}{C} \sum_{n=\text { odd }}^{\infty} \frac{R_{1}^{\prime n}}{n !(2)^{n}} \sum_{r=0}^{n}\left(\begin{array}{l}
n \\
r
\end{array}\right) \frac{(j)^{n-2 r+1}}{a^{2}+\omega^{2}(2 r-n)^{2}} f(\psi) \\
& +\frac{I_{s}}{C} \sum_{n=\text { even }}^{\infty} \frac{R_{1}^{\prime} n}{n !(2)^{n}} \sum_{r=0}^{n}\left(\begin{array}{l}
n \\
r
\end{array}\right) \frac{(j)^{n-2 r}}{a^{2}+\omega^{2}(2 r-n)^{2}} g(\psi) .
\end{aligned}
$$

\section{Calculation for the second-order perturbation term}

$$
\begin{aligned}
V_{02}= & \frac{-I_{s}}{C} \int_{0}^{t} e^{-(t-\tau) / R^{\prime} C} g^{\prime}\left(V_{i}(\tau)-V_{00}(\tau)\right) V_{01}(\tau) d \tau \\
= & \frac{-I_{s} \exp (-a t)}{C} \int_{0}^{t} \exp (a \tau) \omega R_{1}^{\prime} \cos (\omega \tau+\theta)\left(\exp \left(R_{1}^{\prime} \sin (\omega \tau+\theta)\right)-1\right) V_{01}(\tau) d \tau \\
= & \frac{-I_{s} \exp (-a t)}{C} \int_{0}^{t} \frac{\omega R_{1}^{\prime}}{2}(\exp (a \tau+j(\omega \tau+\theta))) \\
& +\exp (a \tau-j(\omega \tau+\theta))\left(\exp \left(R_{1}^{\prime} \sin (\omega \tau+\theta)\right)-1\right) V_{01}(\tau) d \tau .
\end{aligned}
$$

Rewrite the above integral in the following form:

$$
V_{02}=\frac{-I_{s} \exp (-a t)}{C} \omega R_{1}^{\prime}\left[I_{1}+I_{2}-I_{3}\right]
$$

where

$$
\begin{gathered}
I_{1}=\int_{0}^{t} \frac{1}{2} \exp \left(a \tau+R_{1}^{\prime} \sin (\omega \tau+\theta)+j(\omega \tau+\theta)\right) V_{01}(\tau) d \tau \\
I_{2}=\int_{0}^{t} \frac{1}{2} \exp \left(a \tau+R_{1}^{\prime} \sin (\omega \tau+\theta)-j(\omega \tau+\theta)\right) V_{01}(\tau) d \tau \\
I_{3}=\int_{0}^{t} \exp (a \tau) \cos (\omega \tau+\theta) V_{01}(\tau) .
\end{gathered}
$$

Solve the integral $I_{1}$ :

$$
\begin{aligned}
I_{1}= & \int_{0}^{t} \frac{1}{2} \exp \left(a \tau+R_{1}^{\prime} \sin (\omega \tau+\theta)+j(\omega \tau+\theta)\right) \\
& \times\left(\frac{I_{s}}{C} \sum_{n=\text { odd }}^{\infty} \frac{R_{1}^{\prime n}}{n !(2)^{n}} \sum_{r=0}^{n}\left(\begin{array}{l}
n \\
r
\end{array}\right) \frac{(j)^{n-2 r+1}}{a^{2}+\omega^{2}(2 r-n)^{2}} f(\psi)\right) \\
& +\frac{1}{2} \exp \left(a \tau+R_{1}^{\prime} \sin (\omega \tau+\theta)+j(\omega \tau+\theta)\right) \\
& \times\left(\frac{I_{s}}{C} \sum_{n=\text { even }}^{\infty} \frac{R_{1}^{\prime n}}{n !(2)^{n}} \sum_{r=0}^{n}\left(\begin{array}{l}
n \\
r
\end{array}\right) \frac{(j)^{n-2 r}}{a^{2}+\omega^{2}(2 r-n)^{2}} g(\psi)\right) .
\end{aligned}
$$


Solving the integral, we get

$$
\begin{aligned}
& =\frac{I_{s}}{2 C} \sum_{n=\mathrm{odd}}^{\infty} \frac{R_{1}^{\prime n}}{n !(2)^{n}} \sum_{r=0}^{n}\left(\begin{array}{l}
n \\
r
\end{array}\right) \frac{(j)^{n-2 r+1}}{a^{2}+\omega^{2}(2 r-n)^{2}} \frac{a}{\omega} \sum_{m=0}^{\infty} \frac{R_{1}^{\prime m}}{m !(2)^{m}} \sum_{k=0}^{m+1}\left(\begin{array}{c}
m+1 \\
k
\end{array}\right)(-1)^{k-m-1} \\
& *\left(\frac{\exp (a t+j(\omega t+\theta)(2 k-m+1))-\exp (\xi+\theta)}{a / \omega+j(2 k-m+1)}\right) \\
& -\frac{I_{s}}{2 C} \sum_{n=\text { odd }}^{\infty} \frac{R_{1}^{\prime n}}{n !(2)^{n}} \sum_{r=0}^{n}\left(\begin{array}{l}
n \\
r
\end{array}\right) \frac{(j)^{n-2 r+1}}{a^{2}+\omega^{2}(2 r-n)^{2}} \frac{2 r-n}{2} * \sum_{m=0}^{\infty} \frac{R_{1}^{\prime} m}{m !(2)^{m}} \sum_{k=0}^{m}\left(\begin{array}{l}
m \\
k
\end{array}\right)(-1)^{k-m} \\
& \times\left(\frac{\exp (a t+j(\omega t+\theta)(2(k+r)+1-(m+n)))-\exp (j \theta(2(k+r)+1-(m+n)))}{a / \omega+j(2(k+r)-(m+n)+1)}\right. \\
& \left.+\frac{\exp (a t+j(\omega t+\theta)(2(k+r)-1-(m+n)))-\exp (j \theta(2(k+r)-1-(m+n)))}{a / \omega+j(2(k+r)-(m+n)-1)}\right) \\
& +\frac{I_{s}}{2 C} \sum_{n=\mathrm{even}}^{\infty} \frac{R_{1}^{\prime}{ }^{n}}{n !(2)^{n}} \sum_{r=0}^{n}\left(\begin{array}{l}
n \\
r
\end{array}\right) \frac{(j)^{n-2 r}}{a^{2}+\omega^{2}(2 r-n)^{2}}\left(\frac{a}{2}+\frac{\omega(2 r-n)}{2 j}\right) \sum_{m=0}^{\infty} \frac{R_{1}^{\prime}{ }^{m}}{m !(2)^{m}} \sum_{k=0}^{m}\left(\begin{array}{c}
m \\
k
\end{array}\right)(-1)^{k-m} \\
& \times\left(\frac{\exp (a t+j(2 r+2 k-n-m+1)(\omega t+\theta))}{a+j(2 r+2 k-n-m+1)}-\frac{\exp (j(2 r+2 k-n-m+1) \theta)}{a+j(2 r+2 k-n-m+1)}\right) \\
& +\frac{I_{s}}{2 C} \sum_{n=\mathrm{even}}^{\infty} \frac{R_{1}^{\prime n}}{n !(2)^{n}} \sum_{r=0}^{n}\left(\begin{array}{l}
n \\
r
\end{array}\right) \frac{(j)^{n-2 r}}{a^{2}+\omega^{2}(2 r-n)^{2}}\left(\frac{a}{2}-\frac{\omega(2 r-n)}{2 j}\right) \sum_{m=0}^{\infty} \frac{R_{1}^{\prime m}}{m !(2)^{m}} \sum_{k=0}^{m}\left(\begin{array}{c}
m \\
k
\end{array}\right)(-1)^{k-m} \\
& \times\left(\frac{\exp (a t+j(2 r+2 k-n-m-1)(\omega t+\theta))-\exp (j(2 r+2 k-n-m-1) \theta)}{a+j(2 r+2 k-n-m-1)}\right) .
\end{aligned}
$$

The integral $I_{2}$ can be obtained by just replacing the term $j(\omega \tau+\theta)$ by $-j(\omega \tau+\theta)$ in the above result:

$$
\begin{aligned}
= & \frac{I_{s}}{2 C} \sum_{n=\text { odd }}^{\infty} \frac{R_{1}^{\prime n}}{n !(2)^{n}} \sum_{r=0}^{n}\left(\begin{array}{l}
n \\
r
\end{array}\right) \frac{(j)^{n-2 r+1}}{a^{2}+\omega^{2}(2 r-n)^{2}} \frac{a}{\omega} \sum_{m=0}^{\infty} \frac{R_{1}^{\prime m}}{m !(2)^{m}} \sum_{k=0}^{m+1}\left(\begin{array}{c}
m+1 \\
k
\end{array}\right)(-1)^{k-m-1} \\
& *\left(\frac{\exp (a t+j(\omega t+\theta)(2 k-m-1))-\exp (\xi-\theta)}{a / \omega+j(2 k-m-1)}\right) \\
& -\frac{I_{s}}{2 C} \sum_{n=\text { odd }}^{\infty} \frac{R_{1}^{\prime n}}{n !(2)^{n}} \sum_{r=0}^{n}\left(\begin{array}{l}
n \\
r
\end{array}\right) \frac{(j)^{n-2 r+1}}{a^{2}+\omega^{2}(2 r-n)^{2}} \frac{2 r-n}{2} * \sum_{m=0}^{\infty} \frac{R_{1}^{\prime m}}{m !(2)^{m}} \sum_{k=0}^{m}\left(\begin{array}{c}
m \\
k
\end{array}\right)(-1)^{k-m}
\end{aligned}
$$


12 A perturbation-based model for rectifier circuits

$$
\begin{aligned}
& \times\left(\frac{\exp (a t+j(\omega t+\theta)(2(k+r)-1-(m+n)))-\exp (j \theta(2(k+r)-1-(m+n)))}{a / \omega+j(2(k+r)-(m+n)-1)}\right. \\
& \left.+\frac{\exp (a t+j(\omega t+\theta)(2(k+r)+1-(m+n)))-\exp (j \theta(2(k+r)+1-(m+n)))}{a / \omega+j(2(k+r)-(m+n)+1)}\right) \\
& +\frac{I_{s}}{2 C} \sum_{n=\mathrm{even}}^{\infty} \frac{R_{1}^{\prime n}}{n !(2)^{n}} \sum_{r=0}^{n}\left(\begin{array}{l}
n \\
r
\end{array}\right) \frac{(j)^{n-2 r}}{a^{2}+\omega^{2}(2 r-n)^{2}}\left(\frac{a}{2}+\frac{\omega(2 r-n)}{2 j}\right) \sum_{m=0}^{\infty} \frac{R_{1}^{\prime m}}{m !(2)^{m}} \sum_{k=0}^{m}\left(\begin{array}{c}
m \\
k
\end{array}\right)(-1)^{k-m} \\
& \times\left(\frac{\exp (a t+j(2 r+2 k-n-m-1)(\omega t+\theta))}{a+j(2 r+2 k-n-m-1)}-\frac{\exp (j(2 r+2 k-n-m-1) \theta)}{a+j(2 r+2 k-n-m-1)}\right) \\
& +\frac{I_{s}}{2 C} \sum_{n=\mathrm{even}}^{\infty} \frac{R_{1}^{\prime n}}{n !(2)^{n}} \sum_{r=0}^{n}\left(\begin{array}{l}
n \\
r
\end{array}\right) \frac{(j)^{n-2 r}}{a^{2}+\omega^{2}(2 r-n)^{2}}\left(\frac{a}{2}-\frac{\omega(2 r-n)}{2 j}\right) \sum_{m=0}^{\infty} \frac{R_{1}^{\prime m}}{m !(2)^{m}} \sum_{k=0}^{m}\left(\begin{array}{c}
m \\
k
\end{array}\right)(-1)^{k-m} \\
& \times\left(\frac{\exp (a t+j(2 r+2 k-n-m+1)(\omega t+\theta))-\exp (j(2 r+2 k-n-m+1) \theta)}{a+j(2 r+2 k-n-m+1)}\right) .
\end{aligned}
$$

Solving for the integral $I_{3}$, we get

$$
\begin{aligned}
& I_{3}=\int_{0}^{t} \exp (a \tau) \cos (\omega \tau+\theta) V_{01}(\tau) \\
& =\frac{I_{s}}{2 C} \sum_{n=\mathrm{odd}}^{\infty} \frac{R_{1}^{\prime}{ }^{n}}{n !(2)^{n}} \sum_{r=0}^{n}\left(\begin{array}{l}
n \\
r
\end{array}\right) \frac{(j)^{n-2 r+1}}{a^{2}+\omega^{2}(2 r-n)^{2}}\left(\frac{a}{2 j}-\frac{\omega(2 r-n)}{2}\right) \\
& \times\left(\frac{\exp (a t+j(\psi+\omega t+\theta))-\exp (j(\xi+\theta))}{a+j(2 r-n+1) \omega}\right) \\
& -\frac{I_{s}}{2 C} \sum_{n=\text { odd }}^{\infty} \frac{R_{1}^{\prime}{ }^{n}}{n !(2)^{n}} \sum_{r=0}^{n}\left(\begin{array}{l}
n \\
r
\end{array}\right) \frac{(j)^{n-2 r+1}}{a^{2}+\omega^{2}(2 r-n)^{2}}\left(\frac{a}{2 j}+\frac{\omega(2 r-n)}{2}\right) \\
& \times\left(\frac{\exp (a t+j(-\psi+\omega t+\theta))-\exp (j(-\xi+\theta))}{a+j(n-2 r+1) \omega}\right) \\
& +\frac{I_{s}}{2 C} \sum_{n=\text { odd }}^{\infty} \frac{R_{1}^{\prime n}}{n !(2)^{n}} \sum_{r=0}^{n}\left(\begin{array}{l}
n \\
r
\end{array}\right) \frac{(j)^{n-2 r+1}}{a^{2}+\omega^{2}(2 r-n)^{2}}\left(\frac{a}{2 j}-\frac{\omega(2 r-n)}{2}\right) \\
& \times\left(\frac{\exp (a t+j(\psi-\omega t-\theta))-\exp (j(\xi-\theta))}{a+j(n-2 r-1) \omega}\right) \\
& -\frac{I_{s}}{2 C} \sum_{n=\text { odd }}^{\infty} \frac{R_{1}^{\prime} n}{n !(2)^{n}} \sum_{r=0}^{n}\left(\begin{array}{l}
n \\
r
\end{array}\right) \frac{(j)^{n-2 r+1}}{a^{2}+\omega^{2}(2 r-n)^{2}}\left(\frac{a}{2 j}+\frac{\omega(2 r-n)}{2}\right) \\
& \times\left(\frac{\exp (a t-j(\psi+\omega t+\theta))-\exp (-j(\xi+\theta))}{a-j(2 r-n+1) \omega}\right)
\end{aligned}
$$




$$
\begin{aligned}
& +\frac{I_{s}}{2 C} \sum_{n=\mathrm{even}}^{\infty} \frac{R_{1}^{\prime n}}{n !(2)^{n}} \sum_{r=0}^{n}\left(\begin{array}{l}
n \\
r
\end{array}\right) \frac{(j)^{n-2 r}}{a^{2}+\omega^{2}(2 r-n)^{2}}\left(\frac{a}{2}+\frac{w(2 r-n)}{2 j}\right) \\
& \times\left(\frac{\exp (a t+j(\omega t+\theta+\psi))-\exp (j(\theta+\xi))}{a+j(2 r-n+1) \omega}\right) \\
& +\frac{I_{s}}{2 C} \sum_{n=\mathrm{even}}^{\infty} \frac{R_{1}^{\prime n}}{n !(2)^{n}} \sum_{r=0}^{n}\left(\begin{array}{l}
n \\
r
\end{array}\right) \frac{(j)^{n-2 r}}{a^{2}+\omega^{2}(2 r-n)^{2}}\left(\frac{a}{2}-\frac{w(2 r-n)}{2 j}\right) \\
& \times\left(\frac{\exp (a t+j(\omega t+\theta-\psi))-\exp (j(\theta-\xi))}{a+j(n-2 r+1) \omega}\right) \\
& +\frac{I_{s}}{2 C} \sum_{n=\mathrm{even}}^{\infty} \frac{R_{1}^{\prime n}}{n !(2)^{n}} \sum_{r=0}^{n}\left(\begin{array}{l}
n \\
r
\end{array}\right) \frac{(j)^{n-2 r}}{a^{2}+\omega^{2}(2 r-n)^{2}}\left(\frac{a}{2}+\frac{w(2 r-n)}{2 j}\right) \\
& \times\left(\frac{\exp (a t+j(\psi-\omega t-\theta))-\exp (j(\xi-\theta))}{a+j(2 r-n-1) \omega}\right) \\
& +\frac{I_{s}}{2 C} \sum_{n=\mathrm{even}}^{\infty} \frac{R_{1}^{\prime n}}{n !(2)^{n}} \sum_{r=0}^{n}\left(\begin{array}{l}
n \\
r
\end{array}\right) \frac{(j)^{n-2 r}}{a^{2}+\omega^{2}(2 r-n)^{2}}\left(\frac{a}{2}-\frac{w(2 r-n)}{2 j}\right) \\
& \times\left(\frac{\exp (a t-j(\omega t+\theta+\psi))-\exp (-j(\theta+\xi))}{a+j(n-2 r-1) \omega}\right) .
\end{aligned}
$$

Summing up all integrals $I_{1}, I_{2}$, and $I_{3}$ we get the final solution of the second-order perturbation $V_{02}$.

\section{References}

[1] H. K. Khalil, Nonlinear Systems, 2nd ed., Prentice-Hall, New Jersey, 1996.

[2] G. Kriegsmann, An asymptotic theory of rectification and detection, IEEE Transactions on Circuits and Systems 32 (1985), no. 10, 1064-1068.

[3] A. Lieders, Single phase rectifier circuits with CR filters, Electronic Components Application 1 (1979), no. 3, 153-163.

[4] T. C. Scott, R. A. Moore, M. B. Monagan, G. J. Fee, and E. R. Vrscay, Perturbative solutions of quantum mechanical problems by symbolic computation, Journal of Computational Physics 87 (1990), no. 2, 366-395.

[5] A. S. Sedra and K. C. Smith, Microelectronic Circuits, 4th ed., Oxford University Press, Oxford, 2003.

Vipin B. Vats: Division of Electronics and Communication, Netaji Subhas Institute of Technology, New Delhi 110075, India

E-mail address: vipin@ece.nsit.ac.in

H. Parthasarathy: Division of Electronics and Communication, Netaji Subhas Institute of Technology, New Delhi 110075, India

E-mail address: harish@ece.nsit.ac.in 


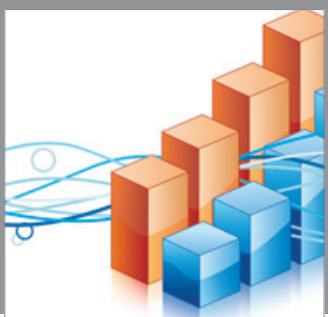

Advances in

Operations Research

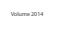

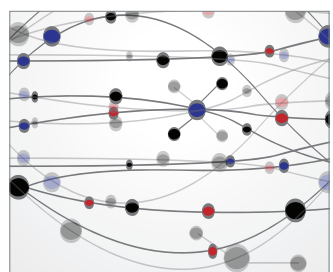

\section{The Scientific} World Journal
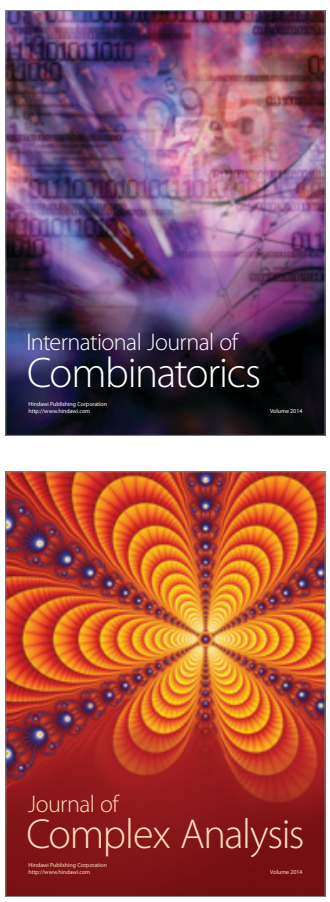

International Journal of

Mathematics and

Mathematical

Sciences
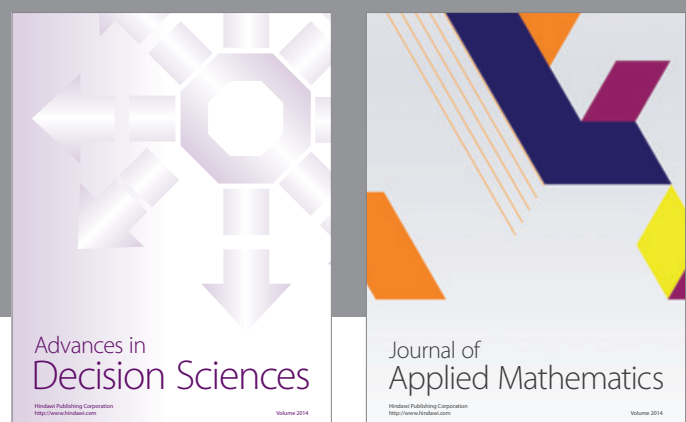

Journal of

Applied Mathematics
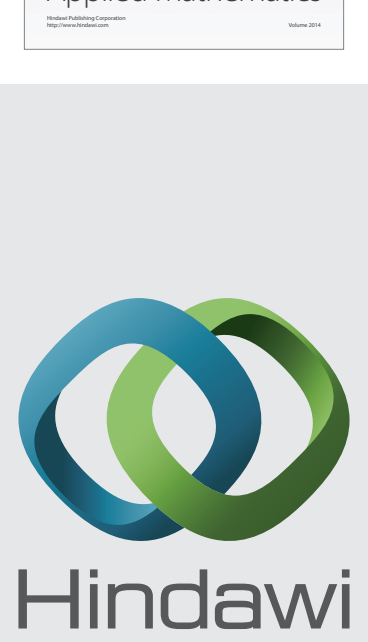

Submit your manuscripts at http://www.hindawi.com
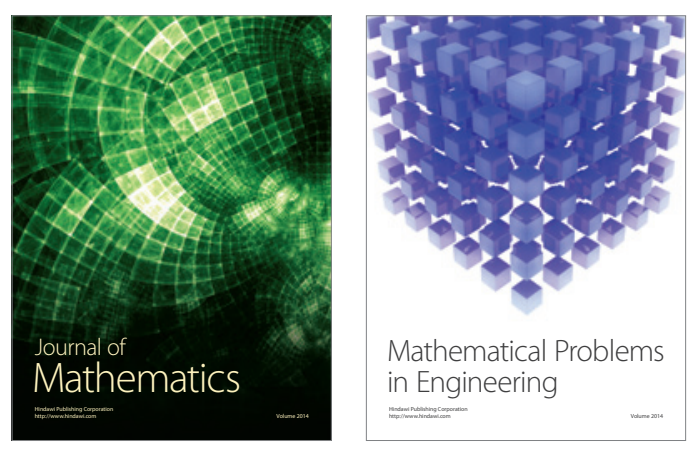

Mathematical Problems in Engineering
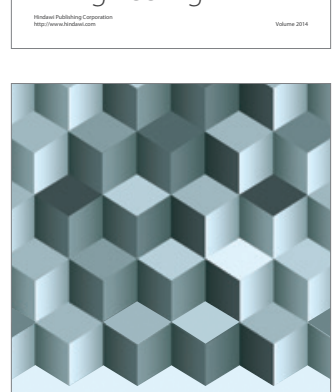

Journal of

Function Spaces
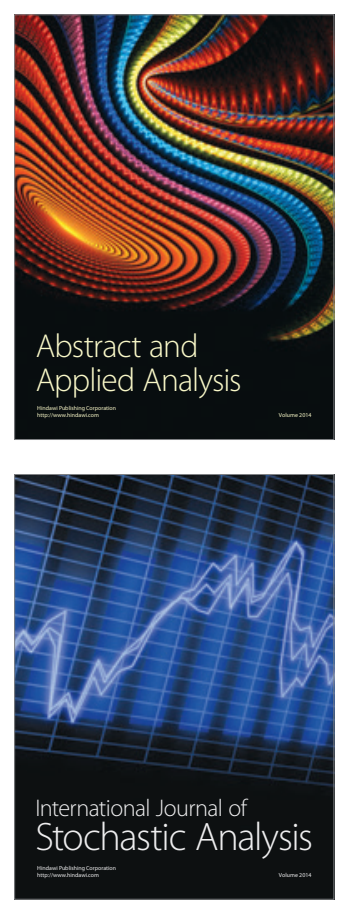

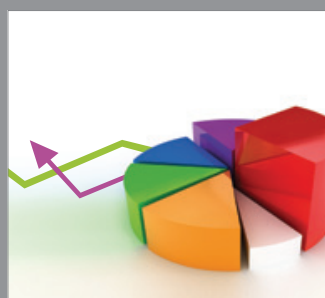

ournal of

Probability and Statistics

Promensencen
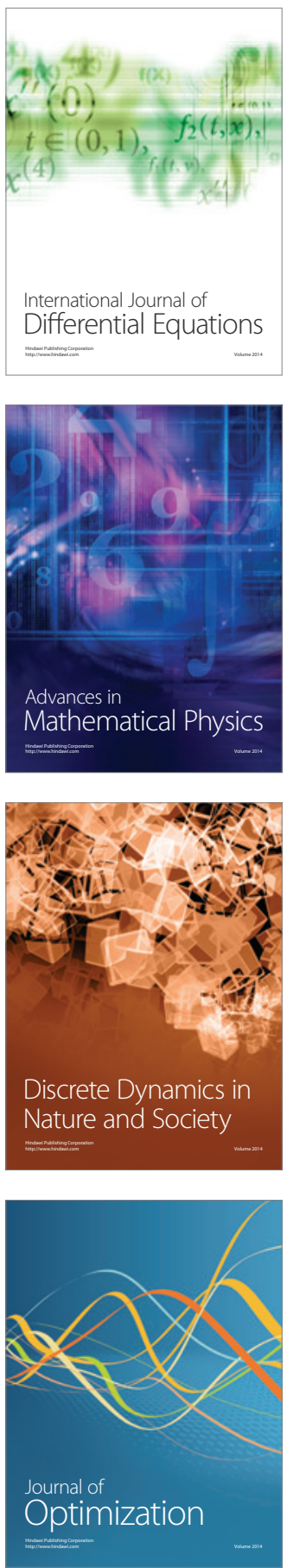